\title{
On the Bodlaender Sequence
}

\author{
Daniele Parisse \\ Airbus Defence and Space GmbH \\ Rechliner Str., 85077 Manching, Germany \\ daniele.parisse@t-online.de
}

\begin{abstract}
We will present two equivalent solutions of the Bodlaender sequence $g: \mathbb{N} \longrightarrow \mathbb{Z}$ first introduced recursively by him et al. and used by them to determine the edge ranking number of the complete graphs. These solutions rely on the binary representation of $m \in \mathbb{N}$ either in the form $m=2^{a_{0}}+\cdots+2^{a_{l}}$ with the strictly decreasing integer sequence $a_{0}>a_{1}>\cdots>a_{l} \geq 0, l \geq 0$, or using the binary expansion of $m=\sum_{k=0}^{n} b_{k} 2^{k}$, where $n \in \mathbb{N}_{0}$ and $b_{0}, \ldots, b_{n} \in\{0,1\}$. In addition, we will determine sharp bounds of the solution and we will give some properties of related sequences such as $a(m):=\frac{1}{3}\left(m^{2}+g(m)\right), m \in \mathbb{N}$, $d(m):=g(m+1)+1, m \in \mathbb{N}_{0}$, and the sequence of the partial sums of $g(m), m \in \mathbb{N}$.
\end{abstract}

Keywords: sequences, divide-and-conquer recurrences, edge ranking number of a graph

MSC: Primary 11B37; Secondary 05C15.

\section{Introduction}

In order to determine the edge ranking number of complete graphs Hans L. Bodlaender et al. introduced in [1, Chapter 7] the following sequence defined recursively for any $m \in \mathbb{N}$ by the rules

$$
\left.\begin{array}{rl}
g(1) & =-1 \\
g(2 m) & =g(m) \\
g(2 m+1) & =g(m+1)+m
\end{array}\right\}
$$

DOI: https://doi.org/10.5592/CO/CCD.2018.08 
with the first few values

$$
(-1,-1,0,-1,2,0,2,-1,6,2,5,0,8,2,6,-1,14,6,11,2,15,5,11,0,20, \ldots) .
$$

(This sequence is not available in the On-Line Encyclopedia of Integer Sequences (OEIS $\left.{ }^{\circledR}\right)[8]$.)

We briefly recall the definition of the edge ranking number of a graph $G$ (for more details we refer to $[1$, Definition 2] or [7, p.1067].) Let $G=(V, E)$ be a (simple) graph and $t$ a positive integer. An edge-t-ranking is an edge coloring $c^{\prime}: E \longrightarrow\{1,2, \ldots, t\}$ such that for any two edges of the same color, every path between them contains an intermediate edge with a larger color value. The edge ranking number denoted by $\chi_{r}^{\prime}(G)$ is the smallest value of $t$ such that the graph $G$ has an edge-t-ranking.

Remark 1.1 In [4, p.1068] and [7, Corollary 4.9] the authors called it Bodlaender function $g$, but since (1.1) is defined only for all $m \in \mathbb{N}$, it is more appropriate to denote it Bodlaender sequence.

Note that for $m-1$ instead of $m$ we obtain from (1.1) $g(2(m-1)+1)=$ $g(2 m-1)=g(m)+m-1$ and therefore $g(2 m-1)-g(2 m)=m-1$ for all $m \in \mathbb{N}$.

The recurrence relation (1.1) is a special case of the general recurrence relation defined for all $m \in \mathbb{N}$

$$
\left.\begin{array}{rl}
f(1) & =\zeta \\
f(2 m) & =\alpha f(m)+g(m) \\
f(2 m+1) & =\gamma f(m)+\delta f(m+1)+h(m)
\end{array}\right\}
$$

with the parameters $\alpha, \gamma, \delta, \zeta \in \mathbb{Z}$ and the integer functions $g, h: \mathbb{N} \longrightarrow \mathbb{Z}$. In our case it is $\alpha=1, \gamma=0, \delta=1, \zeta=-1$ and $g(m)=0, h(m)=m$ for all $m \in \mathbb{N}$.

Eq.(1.2) can also be written for all $m \in \mathbb{N}_{2}:=\{2,3,4, \ldots\}$ as follows

$$
f(m)=a(m) f(\lfloor m / 2\rfloor)+b(m) f(\lceil m / 2\rceil)+c(m), f(1)=\zeta
$$

with

and

$$
a(m):=\frac{\alpha+\gamma}{2}+(-1)^{m} \frac{\alpha-\gamma}{2}, \quad b(m):=\frac{1-(-1)^{m}}{2} \delta
$$

$$
c(m):=\frac{g(m)+h(m)}{2}+(-1)^{m} \frac{g(m)-h(m)}{2} .
$$

Recurrence relations of this form are called (binary) divide-and-conquer recurrences and appear often in computer science, because algorithms based on the technique of divide et impera (divide and conquer) often reduce a problem of size $m$ to 
the solution of two problems of approximately equal sizes $\lfloor m / 2\rfloor$ and $\lceil m / 2\rceil$, where $m=\lfloor m / 2\rfloor+\lceil m / 2\rceil, m \in \mathbb{N}_{0}$. The solutions of the two subproblems are then used to solve the original problem.

A prominent example is given by the problem to sort $m$ records, $m>1$. One method is called mergesort [3, p.79] and consists in dividing the $m$ records into two approximately equal parts, one of size $\lfloor m / 2\rfloor$ and the other of size $\lceil m / 2\rceil$. After each part has been sorted separately by the same method, the records are merged into their final order by doing at most $m-1$ further comparisons. The total number of comparisons performed is at most $f(m)$, where $f(1)=0$ and $f(m)=f(\lfloor m / 2\rfloor)+f(\lceil m / 2\rceil)+m-1, m>1$. This is a special case of (1.3) with $a(m)=b(m)=1, c(m)=m-1$ and $\zeta=0$.

Another well-known example is given by Stern's diatomic sequence (this is the sequence $\underline{\mathrm{A} 002487}$ in the OEIS [8]), defined by $s(1)=1, s(2 m)=s(m)$ and $s(2 m+1)=s(m)+s(m+1)$ for all $m \in \mathbb{N}$, that is $\alpha=\gamma=\delta=\zeta=1$ and $g(m)=h(m)=0$ for all $m \in \mathbb{N}$. Note that the value $s(0)$ has to be 0 , since from the recurrence relation for odd indices for $m=0$ we get $s(1)=s(0)+s(1)$, that is $s(0)=0$.

Adding 1 and starting with the index 0 we get from $g(m)$ the sequence $d(m):=$ $g(m+1)+1, m \in \mathbb{N}_{0}$, satisfying for all $m \geq 1$ the recurrence relation

$$
\left.\begin{array}{rl}
d(0) & =g(1)+1=-1+1=0, d(1)=g(2)+1=0 \\
d(2 m) & =g(2 m+1)+1=g(m+1)+m+1=d(m)+m \\
m+1) & =g(2 m+1+1)+1=g(m+1)+1=d(m)
\end{array}\right\}
$$

This is the sequence A233931 in the OEIS [8] with the first few values

$$
(0,0,1,0,3,1,3,0,7,3,6,1,9,3,7,0,15,7,12,3,16,6,12,1,21,9,16,3, \ldots)
$$

and a special case of (1.2) with $\alpha=1, \gamma=1, \delta=0, \zeta=0, g(m)=m$ and $h(m)=0$.

In this paper we shall determine the solution of (1.1) by writing $m \in \mathbb{N}$ either in the form $m=2^{a_{0}}+\cdots+2^{a_{l}}$ with the strictly decreasing integer sequence $a_{0}>a_{1}>$ $\cdots>a_{l} \geq 0, l \geq 0$, or using the binary expansion of $m=\left(b_{n} \ldots b_{0}\right)_{2}:=\sum_{k=0}^{n} b_{k} 2^{k}$, where $n \in \mathbb{N}_{0}$ and $b_{0}, \ldots, b_{n} \in\{0,1\}$. Moreover, we shall also give sharp lower and upper bounds for $g(m)$.

Finally, we shall explore some properties of the sequence $a(m):=\frac{1}{3}\left(m^{2}+\right.$ $g(m)), m \in \mathbb{N}$, giving the edge ranking number of complete graphs and investigate the sequence of the partial sums of $g(m), m \in \mathbb{N}$, and the sequence $d(m):=$ $g(m+1)+1, m \in \mathbb{N}_{0}$. 


\section{Preliminaries}

We start with a lemma which gives the values of the Bodlaender sequence for some special numbers.

Lemma 2.1 Let $a, b, c \in \mathbb{N}_{0}$, then

$$
\begin{aligned}
& g\left(2^{a}\right)=-1, \quad a \geq 0 \\
& g\left(2^{a}-1\right)=2^{a-1}-2, \quad a \geq 1 \\
& g\left(2^{a}+1\right)=2^{a}-2, \quad a \geq 0 \\
& g\left(2^{a}+2^{b}\right)=2^{a-b}-2, \quad a>b \geq 0 \\
& g\left(3 \cdot 2^{a}\right)=0, \\
& a \geq 0 \\
& g\left(2^{a}+2^{b}-1\right)=2^{a-1}+2^{b-1}+2^{a-b}-3, \quad a>b \geq 1 \\
& g\left(2^{a}+2^{b}+1\right)=2^{a}+2^{b}-2^{a-b-1}-3, \quad a>b \geq 0 \\
& g\left(2^{a}+2^{b}+2^{c}\right)=2^{a-c}+2^{b-c}+2^{a-b-1}-3, \quad a>b>c \geq 0
\end{aligned}
$$

Proof. a) By repeated use of (1.1) we have $g\left(2^{a}\right)=g(1)=-1$.

b) By (1.1) and Eq.(2.1) we have $g\left(2^{a}-1\right)=g\left(2 \cdot\left(2^{a-1}-1\right)+1\right)=g\left(2^{a-1}-1+\right.$ 1) $+2^{a-1}-1=-1+2^{a-1}-1=2^{a-1}-2$.

c) Let $f(a):=g\left(2^{a}+1\right), a \geq 0$, then by (1.1) the sequence $(f(a))_{a \in \mathbb{N}_{0}}$ satisfies the recurrence relation

$$
f(a+1)=g\left(2^{a+1}+1\right)=g\left(2 \cdot 2^{a}+1\right)=g\left(2^{a}+1\right)+2^{a}=f(a)+2^{a}, a \geq 0,
$$

with $f(0)=g(2)=-1$. The solution of this linear first-order recurrence relation can be obtained by backward substitution. After $a$ substitutions it follows $f(a)=$ $f(a-1)+2^{a-1}=f(a-2)+2^{a-2}+2^{a-1}=\cdots=f(0)+2^{0}+2^{1}+\cdots+2^{a-1}=$ $-1+\left(2^{a}-1\right)=2^{a}-2$ and this proves the formula $(2.3)$.

d) By (1.1) we have $g\left(2^{a}+2^{b}\right)=g\left(2^{b}\left(2^{a-b}+1\right)\right)=g\left(2^{a-b}+1\right)=2^{a-b}-2$, where the last equation follows from (2.3).

e) By Eq.(2.4) we have $g\left(3 \cdot 2^{a}\right)=g\left(2^{a+1}+2^{a}\right)=2^{a+1-a}-2=0$.

f) By (1.1) and Eq.(2.4) we have $g\left(2^{a}+2^{b}-1\right)=g\left(2 \cdot\left(2^{a-1}+2^{b-1}-1\right)+1\right)=$ $g\left(2^{a-1}+2^{b-1}-1+1\right)+2^{a-1}+2^{b-1}-1=2^{a-b}-2+2^{a-1}+2^{b-1}-1$ and this is exactly formula (2.6).

g) By (1.1) we have $g\left(2^{a}+2^{b}+1\right)=g\left(2 \cdot\left(2^{a-1}+2^{b-1}\right)+1\right)=g\left(2^{a-1}+2^{b-1}+\right.$ 1) $+2^{a-1}+2^{b-1}$. Let $f(a, b):=g\left(2^{a}+2^{b}+1\right)$, then the above equation means that 
$f(a, b)$ satisfies the recurrence relation

$$
f(a, b)=f(a-1, b-1)+2^{a-1}+2^{b-1},
$$

which can be solved by backward substitution. After $b$ substitutions we obtain $f(a, b)=f(a-b, 0)+2^{a-b}+2^{0}+2^{a-b+1}+2^{1}+\cdots+2^{a-1}+2^{b-1}$. The first term is by $(1.1)$ and $(2.3)$ equal to $f(a-b, 0)=g\left(2^{a-b}+2^{0}+1\right)=g\left(2 \cdot\left(2^{a-b-1}+1\right)\right)=$ $g\left(2^{a-b-1}+1\right)=2^{a-b-1}-2$. The other term is the sum of three geometric series, namely $\left(1+2+\cdots+2^{b-1}\right)+\left(1+2+\cdots+2^{a-1}\right)-\left(1+2+\cdots+2^{a-b-1}\right)=$ $\left(2^{b}-1\right)+\left(2^{a-b}-1\right)-\left(2^{a-b}-1\right)=2^{a}+2^{b}-2^{a-b}-1$. Summing up the two terms it follows $2^{a-b-1}-2+2^{a}+2^{b}-2^{a-b}-1=2^{a}+2^{b}-2^{a-b-1}-3$, as asserted.

h) By (1.1) and Eq.(2.7) we have $g\left(2^{a}+2^{b}+2^{c}\right)=g\left(2^{c} \cdot\left(2^{a-c}+2^{b-c}+1\right)\right)=$ $g\left(2^{a-c}+2^{b-c}+1\right)=2^{a-c}+2^{b-c}+2^{a-b-1}-3$.

The next lemma shows that it is sufficient to consider only the case $a_{l}=0$, that is $m$ is an odd number.

Lemma 2.2 Let $m=2^{a_{0}}+\cdots+2^{a_{l}}$, where $a_{0}>a_{1}>\cdots>a_{l} \geq 0, l \geq 0$, is a strictly decreasing integer sequence, then

for $a_{l} \neq 0$ :

$$
g(m)=g\left(2^{-a_{l}} m\right)
$$

and for $a_{l}=0$ :

$$
g(m)=g\left(2^{-a_{l-1}}(m-1)+1\right)+\left(1-2^{-a_{l-1}}\right)(m-1)
$$

Proof. a) Let $a_{l} \neq 0$, then by (1.1)

$$
\begin{aligned}
g(m) & =g\left(2^{a_{0}}+\cdots+2^{a_{l}}\right)=g\left(2^{a_{l}}\left(2^{a_{0}-a_{l}}+\cdots+2^{a_{l-1}-a_{l}}+1\right)\right) \\
& =g\left(2^{a_{0}-a_{l}}+\cdots+2^{a_{l-1}-a_{l}}+1\right)=g\left(2^{-a_{l}}\left(m-2^{a_{l}}\right)+1\right)=g\left(2^{-a_{l}} m\right)
\end{aligned}
$$

b) Now let $a_{l}=0$, then by (1.1) and since $\sum_{k=0}^{l-1} 2^{a_{k}}=m-1$ we obtain

$$
\begin{aligned}
g(m) & =g\left(2^{a_{0}}+\cdots+2^{a_{l-1}}+1\right)=g\left(2 \cdot\left(2^{a_{0}-1}+\cdots+2^{a_{l-1}-1}\right)+1\right) \\
& =g\left(2^{a_{0}-1}+\cdots+2^{a_{l-1}-1}+1\right)+2^{a_{0}-1}+\cdots+2^{a_{l-1}-1} \\
& =g\left(2 \cdot\left(2^{a_{0}-2}+\cdots+2^{a_{l-1}-2}\right)+1\right)+\frac{1}{2}(m-1) \\
& =g\left(2^{a_{0}-2}+\cdots+2^{a_{l-1}-2}+1\right)+\frac{1}{4}(m-1)+\frac{1}{2}(m-1)
\end{aligned}
$$


Repeating this procedure $a_{l-1}$ times and noting that $\sum_{k=1}^{a_{l-1}} 2^{-k}=\sum_{k=0}^{a_{l-1}} 2^{-k}-$ $1=2 \cdot\left(1-2^{-a_{l-1}-1}\right)-1=1-2^{-a_{l-1}}$ we obtain

$$
\begin{aligned}
g(m) & =g\left(2^{a_{0}-a_{l-1}}+\cdots+2^{a_{l-1}-a_{l-1}}+1\right)+(m-1) \sum_{k=1}^{a_{l-1}} 2^{-k} \\
& =g\left(2^{-a_{l-1}}(m-1)+1\right)+\left(1-2^{-a_{l-1}}\right)(m-1)
\end{aligned}
$$

and this proves Eq.(2.10).

Note that in Eq.(2.10) the argument of $g$ on the left-hand side has $l+1$ summands, whereas on the right-hand side it has only $l$ summands.

\section{$3 \quad$ Main Result}

We can now prove our main result.

Theorem 3.1 Let $m=2^{a_{0}}+\cdots+2^{a_{l}}$, where $a_{0}>a_{1}>\cdots>a_{l} \geq 0, l \geq 0$, is a strictly decreasing integer sequence, then

$$
g(m)=2^{-a_{l}}\left(m-2^{a_{l}}\right)-(l+1)-\sum_{k=1}^{l} 2^{a_{l-k}}\left(\sum_{j=1}^{k-1} 2^{-a_{l-j}-1}\right)
$$

In particular, for $a_{l}=0$

$$
g(m)=m-1-(l+1)-\sum_{k=1}^{l} 2^{a_{l-k}}\left(\sum_{j=1}^{k-1} 2^{-a_{l-j}-1}\right)
$$

Proof. It is sufficient to prove Eq.(3.2), since for $a_{l} \neq 0$ we have $g\left(2^{a_{0}}+\cdots+2^{a_{l}}\right)=$ $g(m)=g\left(2^{-a_{l}} m\right)=g\left(2^{a_{0}-a_{l}}+\cdots+2^{a_{l-1}-a_{l}}+1\right)$ by Eq.(2.9). We obtain Eq.(3.1) by simply writing $a_{k}-a_{l}$ instead of $a_{k}, k=0,1, \ldots, l$ in Eq.(3.2) and noting that $m-1=2^{a_{0}-a_{l}}+\cdots+2^{a_{l-1}-a_{l}}$ and $a_{l-k}-a_{l}-\left(a_{l-j}-a_{l}\right)-1=a_{l-k}-a_{l-j}-1$. Thus the double sum and the term $l+1$ in (3.1) do not change. For the first term we obtain $2^{a_{0}-a_{l}}+\cdots+2^{a_{l-1}-a_{l}}=2^{-a_{l}}\left(2^{a_{0}}+\cdots+2^{a_{l-1}}+2^{a_{l}}-2^{a_{l}}\right)=2^{-a_{l}}\left(m-2^{a_{l}}\right)$ thus obtaining Eq.(3.1).

We now prove the case $a_{l}=0$.

By (2.10) we have

$$
g\left(\sum_{k=0}^{l-1} 2^{a_{k}}+1\right)=g\left(\sum_{k=0}^{l-2} 2^{a_{k}-a_{l-1}-1}+1\right)+\left(1-2^{-a_{l-1}}\right) \sum_{k=1}^{l} 2^{a_{l-k}}
$$


where the argument of $g$ on the left-hand side consists of $l+1$ terms and that on the right-hand side of $l$ terms. Setting $\beta_{k}:=a_{k}-a_{l-1}-1$ for any $k=0,1, \ldots, l-2$, and applying again Eq.(2.10) to the first term in the above equation (3.3), we obtain

$$
g\left(\sum_{k=0}^{l-2} 2^{\beta_{k}}+1\right)=g\left(\sum_{k=0}^{l-3} 2^{\beta_{k}-\beta_{l-2}-1}+1\right)+\left(1-2^{-\beta_{l-2}}\right) \sum_{k=2}^{l} 2^{\beta_{l-k}}
$$

or, in terms of $a_{k}$, noting that for $k=0,1, \ldots, l-3$ we have $\beta_{k}-\beta_{l-2}-1=a_{k}-a_{l-1}-1-\left(a_{l-2}-a_{l-1}-1\right)-1=a_{k}-a_{l-2}-1$

$$
\begin{aligned}
g\left(\sum_{k=0}^{l-2} 2^{a_{k}-a_{l-1}-1}+1\right)= & g\left(\sum_{k=0}^{l-3} 2^{a_{k}-a_{l-2}-1}+1\right) \\
& +\left(1-2^{-\left(a_{l-2}-a_{l-1}-1\right)}\right) \sum_{k=2}^{l} 2^{a_{l-k}-a_{l-1}-1}
\end{aligned}
$$

or, substituting this equation into (3.3)

$$
\begin{aligned}
g\left(\sum_{k=0}^{l-1} 2^{a_{k}}+1\right)= & g\left(\sum_{k=0}^{l-3} 2^{a_{k}-a_{l-2}-1}+1\right)+\left(1-2^{-a_{l-1}}\right) \sum_{k=1}^{l} 2^{a_{l-k}} \\
& +\left(1-2^{-\left(a_{l-2}-a_{l-1}-1\right)}\right) \sum_{k=2}^{l} 2^{a_{l-k}-a_{l-1}-1}
\end{aligned}
$$

Note that the argument of $g$ on the right-hand side has now $l-1$ terms. Repeating this procedure $l-1$ times we finally obtain

$$
\begin{aligned}
g\left(2^{a_{0}}+\cdots+2^{a_{l-1}}+1\right)= & g\left(2^{a_{0}-a_{1}-1}+1\right)+\left(1-2^{-a_{l-1}}\right) \sum_{k=1}^{l} 2^{a_{l-k}} \\
& +\left(1-2^{-\left(a_{l-2}-a_{l-1}-1\right)}\right) \sum_{k=2}^{l} 2^{a_{l-k}-a_{l-1}-1} \\
& +\cdots+\left(1-2^{-\left(a_{1}-a_{2}-1\right)}\right) \sum_{k=l-1}^{l} 2^{a_{l-k}-a_{2}-1}
\end{aligned}
$$


Hence, by Eq.(2.3) and since $g\left(2^{a_{0}-a_{1}-1}+1\right)=2^{a_{0}-a_{1}-1}-2=\left(1-2^{-\left(a_{0}-a_{1}-1\right)}\right)$. $2^{a_{0}-a_{1}-1}-1=\left(1-2^{-\left(a_{0}-a_{1}-1\right)}\right) \sum_{k=l}^{l} 2^{a_{l-k}-a_{1}-1}-1$ it follows

$$
\begin{aligned}
g\left(2^{a_{0}}+\cdots+2^{a_{l-1}}+1\right)= & -1+\left(1-2^{-a_{l-1}}\right) \sum_{k=1}^{l} 2^{a_{l-k}} \\
& +\left(1-2^{-\left(a_{l-2}-a_{l-1}-1\right)}\right) \sum_{k=2}^{l} 2^{a_{l-k}-a_{l-1}-1} \\
& +\cdots+\left(1-2^{-\left(a_{1}-a_{2}-1\right)}\right) \sum_{k=l-1}^{l} 2^{a_{l-k}-a_{2}-1} \\
& +\left(1-2^{-\left(a_{0}-a_{1}-1\right)}\right) \sum_{k=l}^{l} 2^{a_{l-k}-a_{1}-1}
\end{aligned}
$$

A further simplification of the right-hand side of this equation leads to

$$
\begin{aligned}
g\left(\sum_{k=0}^{l-1} 2^{a_{k}}+1\right)= & -1+\sum_{k=1}^{l} 2^{a_{l-k}}-1-\sum_{k=2}^{l} 2^{a_{l-k}-a_{l-1}} \\
& +\sum_{k=2}^{l} 2^{a_{l-k}-a_{l-1}-1}-1-\sum_{k=3}^{l} 2^{a_{l-k}-a_{l-2}} \\
& +\cdots+\sum_{k=l-1}^{l} 2^{a_{l-k}-a_{2}-1}-1-2^{a_{0}-a_{1}}+2^{a_{0}-a_{1}-1}-1 \\
= & \sum_{k=1}^{l} 2^{a_{l-k}}-\sum_{k=2}^{l} 2^{a_{l-k}-a_{l-1}-1}-\sum_{k=3}^{l} 2^{a_{l-k}-a_{l-2}} \\
& -\cdots-\sum_{k=l}^{l} 2^{a_{l-k}-a_{1}-1}-\sum_{k=1}^{l} 1-1
\end{aligned}
$$


and finally

$$
\begin{aligned}
g\left(\sum_{k=0}^{l-1} 2^{a_{k}}+1\right)= & 2^{a_{l-1}}+2^{a_{l-2}}\left(1-2^{-a_{l-1}-1}\right)+2^{a_{l-3}}\left(1-\sum_{j=1}^{2} 2^{-a_{l-j}-1}\right) \\
& +\cdots+2^{a_{0}}\left(1-\sum_{j=1}^{l-1} 2^{-a_{l-j}-1}\right)-(l+1) \\
= & \sum_{k=1}^{l}\left\{2^{a_{l-k}}\left(1-\sum_{j=1}^{k-1} 2^{-a_{l-j}-1}\right)-1\right\}-1
\end{aligned}
$$

and this is Eq.(3.2), since $m-1=\sum_{k=1}^{l} 2^{a_{l-k}}$ and $\sum_{k=1}^{l} 1=l$.

Remark 3.2 Note that $s_{2}(m):=l+1$ is the number of nonzero digits of the binary expansion of $m$ and $m-(l+1)$ is the exponent of the highest power of 2 which divides $m$ ! according to a theorem of Legendre [6, pp.10-12] (for the special case $p=2$ ).

For example, let $m=13=2^{3}+2^{2}+2^{0}$, that is $l=2, a_{0}=3, a_{1}=2, a_{2}=0$, then by $(3.2)$ we have $g(13)=13-1-(2+1)-2^{a_{0}-a_{1}-1}=9-2^{3-2-1}=8$.

A consequence of Theorem 3.1 is the

Proposition 3.3 For all $m \in \mathbb{N}$ we have

$$
-1 \leq g(m) \leq m-1
$$

and, therefore, for all $m \in \mathbb{N}_{0}$

$$
0 \leq d(m) \leq m+1
$$

Proof. Let $m=2^{a_{0}}+\cdots+2^{a_{l}}$, where $a_{0}>a_{1}>\cdots>a_{l} \geq 0, l \geq 0$, is a strictly decreasing integer sequence, then $l+1$ and the double sum in (3.1) are always $\geq 0$ and therefore $g(m) \leq 2^{-a_{l}}\left(m-2^{a_{l}}\right)=2^{-a_{l}} m-1 \leq m-1$, since $2^{a_{l}} \geq 1$. This proves the upper bound.

In order to prove that -1 is a lower bound we note first that by Eq.(2.1) this value is attained for $m=2^{n}, n \geq 0$, and secondly by Eq.(2.9) it is sufficient to consider only odd numbers $m$, that is $a_{l}=0$. As shown in the proof of Theorem 3.1 the formula (3.2) can be written as

$$
g(m)=\sum_{k=1}^{l}\left\{2^{a_{l-k}}\left(1-\sum_{j=1}^{k-1} 2^{-a_{l-j}-1}\right)-1\right\}-1,
$$


therefore we have to prove that

$$
2^{a_{l-k}}\left(1-\sum_{j=1}^{k-1} 2^{-a_{l-j}-1}\right)-1=2^{a_{l-k}}-1-\sum_{j=1}^{k-1} 2^{a_{l-k}-a_{l-j}-1} \geq 0 .
$$

This can be proved as follows: By definition, $a_{l-j} \geq j$ for any $j=0,1, \ldots, l$ or $-a_{l-j} \leq-j$ and, therefore, $\sum_{j=1}^{k-1} 2^{-a_{l-j}} \leq \sum_{j=1}^{k-1} 2^{-j}=1-2^{-(k-1)}$. It follows $1-\frac{1}{2} \sum_{j=1}^{k-1} 2^{-a_{l-j}} \geq 1-\frac{1}{2} \cdot\left(1-\frac{1}{2^{k-1}}\right)=\frac{1}{2}+\frac{1}{2^{k}} \geq \frac{1}{2^{k}}$ and, multiplying both sides by $2^{a_{l-k}}$ and adding to both sides -1 , we obtain $2^{a_{l-k}}\left(1-\sum_{j=1}^{k-1} 2^{-a_{l-j}-1}\right)-1 \geq$ $2^{a_{l-k}-k}-1 \geq 0$, since $a_{l-k}-k \geq 0$ for any $k=1, \ldots, l$. This proves Eq.(3.4). Finally, the double inequality (3.5) follows immediately from the definition of the sequence $(d(m))_{m \in \mathbb{N}_{0}}$.

By means of $h(m):=g(m+1), m \in \mathbb{N}_{0}, h(0)=g(1)=-1$, we can give another representation of the solution (3.1) which will lead to an improvement of Proposition 3.3 .

Proposition 3.4 The sequence $(h(m))_{m \in \mathbb{N}_{0}}$ satisfies for all $m \geq 1$ the recurrence relation

$$
\left.\begin{array}{rl}
h(0) & =-1(h(1)=-1) \\
h(2 m) & =h(m)+m \\
h(2 m+1) & =h(m)
\end{array}\right\}
$$

Proof. By (1.1) it follows $h(0)=g(1)=-1$ and $h(1)=g(2)=-1$. Further

$$
\begin{aligned}
h(2 m) & =g(2 m+1)=g(m+1)+m=h(m)+m \\
h(2 m+1) & =g(2 m+2)=g(m+1)=h(m)
\end{aligned}
$$

and this proves the proposition.

Note that (3.6) is a special case of (1.2) with $\alpha=1, \gamma=1, \delta=0, \zeta=-1, g(m)=$ $m$ and $h(m)=0$.

Using the binary expansion of $m=\left(b_{n} \ldots b_{0}\right)_{2}$, where $b_{0}, \ldots, b_{n} \in\{0,1\}$, we can derive an alternative solution of (1.1).

Theorem 3.5 Let $m=\left(b_{n} \ldots b_{0}\right)_{2} \in \mathbb{N}_{0}$, then the sequence $(h(m))_{m \in \mathbb{N}_{0}}$ satisfies the recurrence relation

$$
h\left(\left(b_{n} \ldots b_{0}\right)_{2}\right)=h\left(\left(b_{n} \ldots b_{1}\right)_{2}\right)+\left(1-b_{0}\right) \cdot\left(b_{n} \ldots b_{1}\right)_{2}
$$


with the solution

$$
g\left(\left(b_{n} \ldots b_{0}\right)_{2}+1\right)=h\left(\left(b_{n} \ldots b_{0}\right)_{2}\right)=-1+\sum_{k=0}^{n-1}\left(1-b_{k}\right)\left(b_{n} \ldots b_{k+1}\right)_{2}
$$

and, therefore, for all $m \in \mathbb{N}_{0}$

$$
d\left(\left(b_{n} \ldots b_{0}\right)_{2}\right)=g\left(\left(b_{n} \ldots b_{0}\right)_{2}+1\right)+1=\sum_{k=0}^{n-1}\left(1-b_{k}\right)\left(b_{n} \ldots b_{k+1}\right)_{2}
$$

Proof. Let $m=\left(b_{n} \ldots b_{0}\right)_{2} \in \mathbb{N}_{0}$, then

$$
\begin{aligned}
h\left(\left(b_{n} \ldots b_{0}\right)_{2}\right) & =b_{0} h\left(\left(b_{n} \ldots b_{1}\right)_{2}\right)+\left(1-b_{0}\right)\left(h\left(\left(b_{n} \ldots b_{1}\right)_{2}\right)+\left(b_{n} \ldots b_{1}\right)_{2}\right) \\
& =h\left(\left(b_{n} \ldots b_{1}\right)_{2}\right)+\left(1-b_{0}\right) \cdot\left(b_{n} \ldots b_{1}\right)_{2}
\end{aligned}
$$

since for $b_{0}=0$, that is $m$ is even, we have $m / 2=\left(b_{n} \ldots b_{1}\right)_{2}$ and by $(3.6) h(m)=$ $h(m / 2)+m / 2=h\left(\left(b_{n} \ldots b_{1}\right)_{2}\right)+\left(b_{n} \ldots b_{1}\right)_{2}$ and for $b_{0}=1$, that is $m$ is odd, we have $(m-1) / 2=\left(b_{n} \ldots b_{1}\right)_{2}$ and by $(3.6) h(m)=h((m-1) / 2)+m / 2=h\left(\left(b_{n} \ldots b_{1}\right)_{2}\right)$. This proves Eq.(3.7).

Repeating this procedure $n-1$ times we finally obtain $h\left(\left(b_{n} \ldots b_{0}\right)_{2}\right)=h\left(b_{n}\right)+$ $\sum_{k=0}^{n-1}\left(1-b_{k}\right)\left(b_{n} \ldots b_{k+1}\right)_{2}$ and this proves Eq.(3.8), since $b_{n}=1$ and $h(1)=-1$. Finally, by definition it follows Eq.(3.9).

For example, let $m=10=(1010)_{2}$, that is $n=3, b_{0}=0, b_{1}=1, b_{2}=0$ and $b_{3}=1$. Then

$$
\begin{aligned}
g(11) & =-1+\sum_{k=0}^{2}\left(1-b_{k}\right)\left(b_{3} \ldots b_{k+1}\right)_{2} \\
& =-1+\left(1-b_{0}\right)\left(b_{3} b_{2} b_{1}\right)_{2}+\left(1-b_{1}\right)\left(b_{3} b_{2}\right)_{2}+\left(1-b_{2}\right)\left(b_{3}\right)_{2} \\
& =-1+(101)_{2}+0 \cdot(10)_{2}+1=-1+5+0+1=5 .
\end{aligned}
$$

From Eq.(3.8) one can deduce that $h$ attains its greatest value at $m=2^{n}=$ $(10 \ldots 0)_{2}$. In this case we have $b_{k}=0$ for all $k=0,1, \ldots, n-1$. Hence $1-b_{k}=1$ for all $k=0,1, \ldots, n-1$. Therefore $g\left(2^{n}+1\right)=h\left(2^{n}\right)=-1+\sum_{k=0}^{n-1}\left(b_{n} \ldots b_{k+1}\right)_{2}=-1+$ $\sum_{k=0}^{n-1} 2^{n-1-k}=-1+2^{n}-1=2^{n}-2$ (see also Eq.(2.3)). Hence, $2^{n}+1-\left(2^{n}-2\right)=3$, that is for all $m \geq 2$ we have $m-g(m) \geq 3$.

Similarly, $h$ attains its smallest value at $m=2^{n}-1=(11 \ldots 1)_{2}$. In this case we 
have $b_{k}=1$ for all $k=0,1, \ldots, n-1$ and hence $1-b_{k}=0$ for all $k=0,1, \ldots, n-1$. Therefore $g\left((1 \ldots 1)_{2}+1\right)=g\left(2^{n}\right)=-1$ (see also Eq.(2.1)). Hence, $g(m) \geq-1$ for all $m \geq 1$.

Thus, we have shown the desired improvement of Proposition 3.3.

Proposition 3.6 For all $m \in \mathbb{N}_{2}$ we have

$$
-1 \leq g(m) \leq m-3
$$

where both bounds are sharp, since for $m=2^{n}, n \geq 0$, it is $g(m)=-1$ and for $m=2^{n}+1, n \geq 0$, it is $g(m)=m-3$.

Therefore, for all $m \in \mathbb{N}$ we have

$$
0 \leq d(m) \leq m-1
$$

\section{Some Consequences}

In [1, Theorem 25] Bodlaender et al. showed that the edge ranking number of the complete graphs on $p$ vertices, $p \in \mathbb{N}$, is given by $\chi_{r}^{\prime}\left(K_{p}\right)=a(p)$, where $a(p):=$ $\frac{1}{3}\left(p^{2}+g(p)\right)$, and in [7, Theorem 7, Corollary 8] Lin, Juan and Wang showed that the edge ranking number of the Sierpiński graphs is given by $\chi_{r}^{\prime}\left(S_{p}^{n}\right)=n \chi_{r}^{\prime}\left(K_{p}\right)=$ $\frac{n}{3}\left(p^{2}+g(p)\right), n, p \in \mathbb{N}_{2}$. (For a definition of the Sierpiński graphs we refer the reader to the seminal paper of Klavžar and Milutinovič [5] and to the survey paper on Sierpiński-type graphs by Hinz, Klavžar and Zemljič [4].)

Proposition 4.1 The sequence $a(m):=\frac{1}{3}\left(m^{2}+g(m)\right), m \in \mathbb{N}$, satisfies for all $m \in \mathbb{N}$ the recurrence relation

$$
\left.\begin{array}{rl}
a(1) & =0 \\
a(2 m) & =a(m)+m^{2} \\
a(2 m+1) & =a(m+1)+m(m+1)
\end{array}\right\}
$$

In particular,

$$
3 \mid\left(m^{2}+g(m)\right) .
$$

Note that (4.1) is a special case of (1.2) with $\alpha=1, \gamma=0, \delta=1, \zeta=0, g(m)=$ $m^{2}$ and $h(m)=m(m+1)$. 
Proof. By definition $a(1)=\frac{1}{3}\left(1^{2}+g(1)\right)=\frac{1}{3}(1-1)=0$ and by (1.1) it follows that

$$
\begin{aligned}
a(2 m) & =\frac{4 m^{2}+g(2 m)}{3}=\frac{m^{2}+g(m)+3 m^{2}}{3}=a(m)+m^{2} \\
a(2 m+1) & =\frac{(2 m+1)^{2}+g(2 m+1)}{3}=\frac{4 m^{2}+4 m+1+g(m+1)+m}{3} \\
& =\frac{(m+1)^{2}+g(m+1)+3 m^{2}+3 m}{3}=a(m+1)+m(m+1)
\end{aligned}
$$

This proves (4.1) and shows that $a(m) \in \mathbb{N}_{0}$, thus proving (4.2).

Note that for $m-1$ instead of $m$ in Eq.(4.1) we obtain $a(2(m-1)+1)=$ $a(2 m-1)=a(m)+(m-1) m$ and therefore $a(2 m)-a(2 m-1)=m^{2}-m(m-1)=m$ for all $m \in \mathbb{N}$.

The first few values of $(a(m))_{m \in \mathbb{N}}$ (not available in the OEIS [8]) are

$$
(0,1,3,5,9,12,17,21,29,34,42,48,59,66,77,85,101,110,124,134, \ldots) .
$$

The next lemma gives the values of this sequence for some special numbers.

Lemma 4.2 Let $n \in \mathbb{N}_{0}$, then

$$
\begin{array}{rlrl}
a\left(2^{n}\right) & =\frac{4^{n}-1}{3} & \\
a\left(2^{n}-1\right) & =\frac{4^{n}-1}{3}-2^{n-1}, & & n \geq 1 \\
a\left(2^{n}+1\right) & =\frac{4^{n}-1}{3}+2^{n} &
\end{array}
$$

Proof. By definition and using Eqs.(2.1), (2.2) and (2.3), we have $a\left(2^{n}\right)=\frac{1}{3}\left(4^{n}+\right.$ $\left.g\left(2^{n}\right)\right)=\frac{1}{3}\left(4^{n}-1\right), a\left(2^{n}-1\right)=\frac{1}{3}\left(\left(2^{n}-1\right)^{2}+g\left(2^{n}-1\right)\right)=\frac{1}{3}\left(4^{n}-1\right)-2^{n-1}=$ $a\left(2^{n}\right)-2^{n-1}$ and $a\left(2^{n}+1\right)=\frac{1}{3}\left(\left(2^{n}+1\right)^{2}+g\left(2^{n}+1\right)\right)=\frac{1}{3}\left(4^{n}-1\right)+2^{n}=a\left(2^{n}\right)+2^{n}$.

We notice that (4.3) is the sequence $\underline{\mathrm{A} 002450},(4.5)$ is the sequence $\underline{\mathrm{A} 079319}$, whereas the sequence (4.4) is not available in the OEIS [8].

Note that for $m=2^{n}, n \geq 0$, we have $a(m)=\frac{1}{3}\left(m^{2}-1\right)$ and for $m=2^{n}+1, n \geq$ 0 , we have $a(m)=\frac{1}{3}\left(m^{2}+m-3\right)$. By Proposition 3.6 we obtain in this way

Corollary 4.3 For all $m \in \mathbb{N}_{2}$ we have

$$
\frac{m^{2}-1}{3} \leq a(m) \leq \frac{m^{2}+m-3}{3},
$$

where both bounds are sharp. 
To conclude we mention some properties of the sequence of the partial sums of $g(m)$.

Proposition 4.4 Let $t(m):=\sum_{k=1}^{m} g(k), m \in \mathbb{N}$, then

$$
\left.\begin{array}{rl}
t(1) & =-1 \\
t(2 m) & =2 t(m)+\left(\begin{array}{c}
m \\
2
\end{array}\right) \\
t(2 m+1) & =t(m)+t(m+1)+\left(\begin{array}{c}
m+1 \\
2
\end{array}\right)
\end{array}\right\}
$$

Note that (4.7) is a special case of (1.2) with $\alpha=2, \gamma=1, \delta=1, \zeta=-1, g(m)=\left(\begin{array}{l}n \\ 2\end{array}\right)$ and $h(m)=\left(\begin{array}{c}m+1 \\ 2\end{array}\right)$.

Proof. It is $t(1)=g(1)=-1$ and by definition we have

$$
\begin{aligned}
t(2 m) & =\sum_{k=1}^{2 m} g(k)=\sum_{k=1}^{m} g(2 k)+\sum_{k=1}^{m-1} g(2 k+1)+g(1) \\
& =\sum_{k=1}^{m} g(k)+\sum_{k=1}^{m-1}(g(k+1)+k)+g(1) \\
& =t(m)+\sum_{k=1}^{m-1} g(k+1)+\sum_{k=1}^{m-1} k+g(1) \\
& =t(m)+t(m)-g(1)+\left(\begin{array}{c}
m \\
2
\end{array}\right)+g(1)=2 t(m)+\left(\begin{array}{c}
m \\
2
\end{array}\right)
\end{aligned}
$$

Similarly,

$$
\begin{aligned}
t(2 m+1) & =\sum_{k=1}^{2 m+1} g(k)=\sum_{k=1}^{m} g(2 k)+\sum_{k=1}^{m} g(2 k+1)+g(1) \\
& =\sum_{k=1}^{m} g(k)+\sum_{k=1}^{m} g(k+1)+\sum_{k=1}^{m} k+g(1) \\
& =t(m)+t(m+1)-g(1)+\left(\begin{array}{c}
m+1 \\
2
\end{array}\right)+g(1) \\
& =2 t(m)+\left(\begin{array}{c}
m+1 \\
2
\end{array}\right)
\end{aligned}
$$


and this proves (4.7).

The first few values of $(t(m))_{m \in \mathbb{N}}$ (not available in the OEIS [8]) are

$$
(-1,-2,-2,-3,-1,-1,1,0,6,8,13,13,21,23,29,28,42,48,59,61,76, \ldots) .
$$

The next lemma gives the values of this sequence for some special numbers.

Lemma 4.5 Let $n \in \mathbb{N}_{0}$, then

$$
\begin{array}{rlrl}
t\left(2^{n}\right) & =\frac{4^{n}-5 \cdot 2^{n}-n \cdot 2^{n}}{4} & & \\
t\left(2^{n}-1\right) & =\frac{4^{n}-5 \cdot 2^{n}-n \cdot 2^{n}}{4}+1, & & n \geq 1 \\
t\left(2^{n}+1\right) & =\frac{4^{n}-5 \cdot 2^{n}-n \cdot 2^{n}}{4}+2^{n}-2, &
\end{array}
$$

Proof. a) Let $f(n):=t\left(2^{n}\right), n \geq 0$, then by (4.7) the sequence $(f(n))_{n \in \mathbb{N}_{0}}$ satisfies the recurrence relation

$$
f(n+1)=t\left(2^{n+1}\right)=t\left(2 \cdot 2^{n}\right)=2 t\left(2^{n}\right)+\left(\begin{array}{c}
2^{n} \\
2
\end{array}\right)=2 f(n)+2^{n-1}\left(2^{n}-1\right), n \geq 0,
$$

with $f(0)=t(1)=-1$. The solution of this linear first-order recurrence relation can be obtained again by backward substitution. After $n$ substitutions it follows

$$
\begin{aligned}
f(n) & =2 f(n-1)+2^{n-2}\left(2^{n-1}-1\right) \\
& =2\left(2 f(n-2)+2^{n-3}\left(2^{n-2}-1\right)\right)+2^{n-2}\left(2^{n-1}-1\right) \\
& =2^{2} f(n-2)+2 \cdot 2^{n-3}\left(2^{n-2}-1\right)+2^{n-2}\left(2^{n-1}-1\right) \\
& =\cdots \cdots \cdots \cdots \\
& =2^{n} f(0)+\sum_{k=0}^{n-1} 2^{k} \cdot 2^{n-2-k} \cdot\left(2^{n-1-k}-1\right) \\
& =-2^{n}+2^{n-2}\left(2^{n}-1-n\right)=\frac{4^{n}-5 \cdot 2^{n}-n \cdot 2^{n}}{4}
\end{aligned}
$$

and this proves (4.8).

b) By definition and using Eqs.(4.8) and (2.1) we get $t\left(2^{n}-1\right)=\sum_{k=1}^{2^{n}-1} g(k)=$ $\sum_{k=1}^{2^{n}} g(k)-g\left(2^{n}\right)=t\left(2^{n}\right)-(-1)=\frac{1}{4}\left(4^{n}-5 \cdot 2^{n}-n \cdot 2^{n}\right)+1$ and this proves (4.9). c) By definition and using Eqs.(4.8) and (2.3) we get $t\left(2^{n}+1\right)=\sum_{k=1}^{2^{n}+1} g(k)=$ 
$\sum_{k=1}^{2^{n}} g(k)+g\left(2^{n}+1\right)=t\left(2^{n}\right)+2^{n}-2=\frac{1}{4}\left(4^{n}-5 \cdot 2^{n}-n \cdot 2^{n}\right)+2^{n}-2$ and this proves (4.10).

Note that for $m=2^{n}, n \geq 0$, that is $n=\log _{2}(m)$, we obtain from (4.8) the sequence $\underline{t}(m):=\frac{m}{4}\left(m-5-\log _{2}(m)\right), m \in \mathbb{N}$. Numerical results suggest that $\underline{t}(m)$ is a lower bound of $t(m)$, that is $\underline{t}(m) \leq t(m), m \in \mathbb{N}$.

An upper bound $\bar{t}(m)$ for $t(m)$ could be obtained from (3.10) as follows: It is $g(k) \leq k-3$ for all $k \in \mathbb{N}_{2}$ and, therefore, $t(m)=g(1)+\sum_{k=2}^{m} g(k) \leq-1+\sum_{k=2}^{m}(k-$ $3)=-1+\sum_{k=2}^{m} k-3 \sum_{k=2}^{m} 1=-1+\left(\begin{array}{c}m+1 \\ 2\end{array}\right)-1-3(m-1)=\frac{1}{2}\left(m^{2}-5 m+2\right)=: \bar{t}(m)$. Since $\frac{1}{2}\left(1^{2}-5 \cdot 1+2\right)=-1=t(1)$ we obtain

$$
\underline{t}(m) \leq t(m) \leq \bar{t}(m), m \in \mathbb{N} .
$$

We notice that $\bar{t}(m+4)=\underline{A 034856}(m), m \in \mathbb{N}$.

Remark 4.6 All the sequences encountered so far, namely $g(m), d(m):=g(m+$ $1)+1, h(m):=g(m+1), a(m):=\frac{1}{3}\left(m^{2}+g(m)\right)$ and $t(m):=\sum_{k=1}^{m} g(k)$ are of divide-and-conquer type, that is their ordinary generating functions defined as the power series $G(s):=\sum_{m=1}^{\infty} g(m) s^{m}, D(s):=\sum_{m=0}^{\infty} d(m) s^{m}, H(s):=\sum_{m=0}^{\infty} h(m) s^{m}$, $A(s):=\sum_{m=1}^{\infty} a(m) s^{m}$ and $T(s):=\sum_{m=1}^{\infty} t(m) s^{m}$ satisfy a functional equation known as Mahlerian equation (cf.[2])

$$
a_{0}(s) F(s)+a_{1}(s) F\left(s^{2}\right)+\cdots+a_{n}(s) F\left(s^{2^{n}}\right)=f(s)
$$

in which $n \in \mathbb{N}, f(s)$ is a formal series and $a_{0}(s), a_{1}(s), \ldots, a_{n}(s)$ are polynomials not all zero. If $f(s)=0$, then the solution of (4.11) is said to be a Mahlerian series.

Indeed, applying standard generating function techniques to the recurrence relations from Eqs.(1.1), (1.4), (3.6), (4.1) and (4.7) we have the functional equations

$$
\begin{aligned}
& G(s)=\left(1+\frac{1}{s}\right) G\left(s^{2}\right)+\frac{s^{3}}{\left(1-s^{2}\right)^{2}} \\
& D(s)=(1+s) D\left(s^{2}\right)+\frac{s^{2}}{\left(1-s^{2}\right)^{2}} \\
& H(s)=(1+s) H\left(s^{2}\right)+\frac{s^{2}}{\left(1-s^{2}\right)^{2}} \\
& A(s)=\left(1+\frac{1}{s}\right) A\left(s^{2}\right)+\frac{s^{2}}{(1+s)(1-s)^{3}} \\
& T(s)=s\left(1+\frac{1}{s}\right)^{2} T\left(s^{2}\right)+\frac{s^{3}}{(1-s)\left(1-s^{2}\right)^{2}}
\end{aligned}
$$


and the relations

$$
D(s)=\frac{G(s)}{s}+\frac{1}{1-s}, H(s)=\frac{G(s)}{s}, A(s)=\frac{1}{3}\left(G(s)+\frac{t(1+s)}{(1-s)^{3}}\right), T(s)=\frac{G(s)}{1-s} .
$$

\section{Acknowledgments}

The author would like to thank Andreas M. Hinz for careful reading of the manuscript, Sandi Klavžar for some advices and an anonymous referee who helped to improve the paper with useful remarks.

\section{References}

[1] H. Bodlaender, J. S. Deogun, K. Jansen, T. Kloks, D. Kratsch, H. Müller and Zs. Tuza, Ranking of graphs, SIAM J. Discrete Mathematics 11 (1998) 168-181.

[2] Ph. Dumas, Algebraic aspects of $B$-regular series, in: Automata, languages and programming (Lund, 1993), Lectures Notes in Comput. Sci. 700, SpringerVerlag, Berlin (1993) 457-468.

[3] R. L. Graham, D. E. Knuth, O. Patashnik, Concrete Mathematics, AddisonWesley, Reading, 1990.

[4] A. M. Hinz, S. Klavžar, S. S. Zemljič, A survey and classification of Sierpińskitype graphs, Discrete Appl. Math. 217 (2017) 565-600.

[5] S. Klavžar, U. Milutinovič, Graphs $t(n, k)$ and a variant of the Tower of Hanoi problem, Czechoslovak Math. J. 47 (122) (1997) 95-104.

[6] A. M. Legendre, Theórie des nombres, Tome I, Troisième Édition, Firmin Didot frères, Paris, 1830.

[7] Y. L. Lin, J. S. Juan, Y. L. Wang, Finding the edge ranking number through vertex partitions, Discrete Appl. Math. 161 (2013) 1067-1071.

[8] OEIS Foundation Inc. (2011), The On-Line Encyclopedia of Integer Sequences, https://oeis.org/ 
